Document downloaded from:

http://hdl.handle.net/10251/37481

This paper must be cited as:

Ballester Beltrán, J.; Cantini, M.; Lebourg, MM.; Rico Tortosa, PM.; Moratal Pérez, D.; García, AJ.; Salmerón Sánchez, M. (2012). Effect of topological cues on material-driven fibronectinfibrillogenesis and cell differentiation. Journal of Materials Science: Materials in Medicine. 23(1):195-204. doi:10.1007/s10856-011-4532-z.

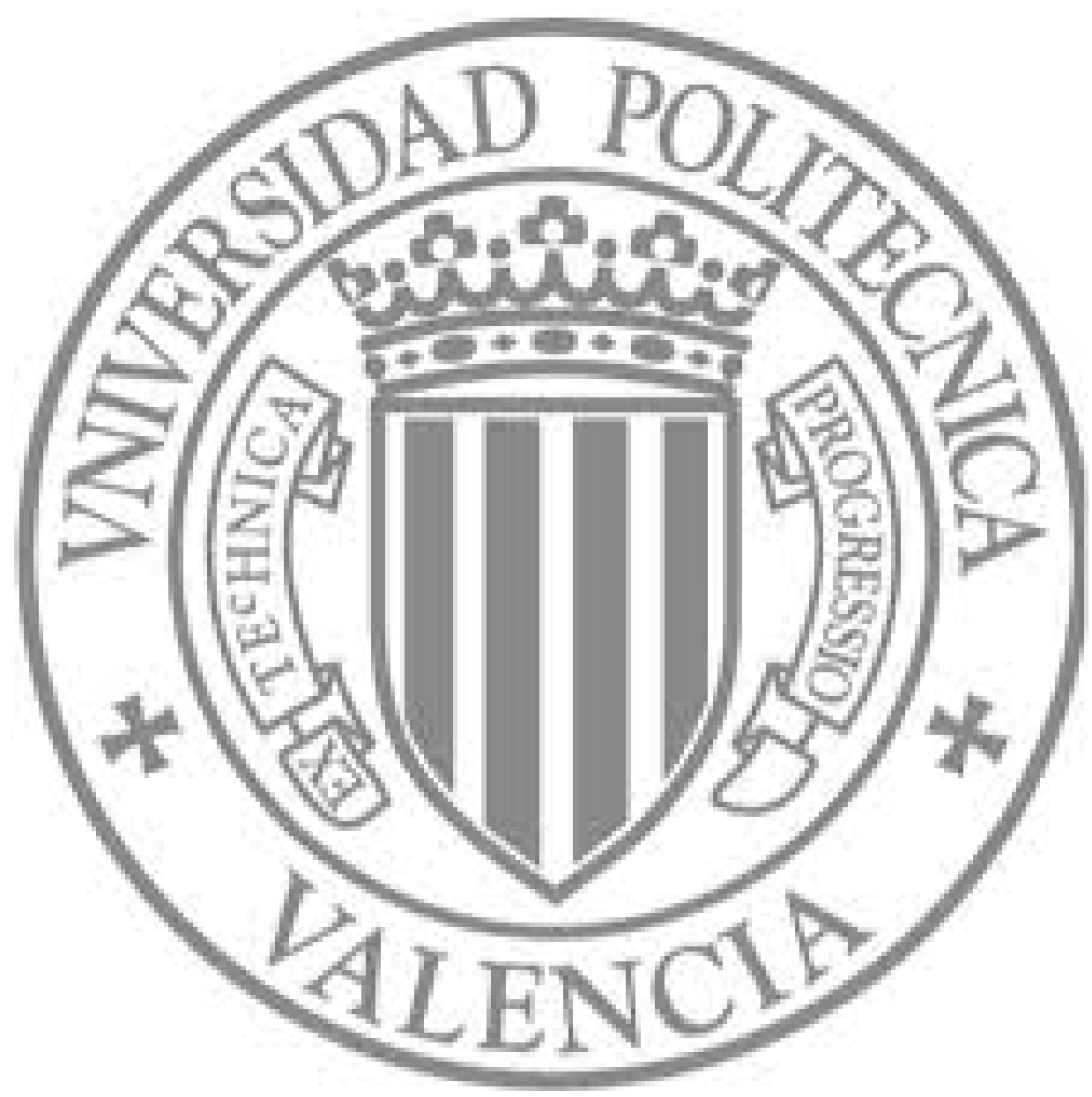

The final publication is available at

http://dx.doi.org/10.1007/s10856-011-4532-z

Copyright

Springer Verlag (Germany) 


\section{Effect of topological cues on material-driven fibronectin fibrillogenesis and cell differentiation}

José Ballester-Beltrán $^{1 *}$, Marco Cantini ${ }^{1 *}$, Myriam Lebourg ${ }^{1,2 *}$, Patricia Rico ${ }^{1,2}$,

David Moratal $^{1}$, Andrés J. García ${ }^{3}$, Manuel Salmerón-Sánchez ${ }^{1,2 \dagger}$

${ }^{1}$ Center for Biomaterials and Tissue Engineering, Universitat Politècnica de València, Spain

${ }^{2}$ CIBER de Bioingeniería, Biomateriales y Nanomedicina, Valencia, Spain

${ }^{3}$ Woodruff School of Mechanical Engineering and Petit Institute for Bioengineering and Bioscience, Georgia Insitute of Technology, Atlanta, GA, United States of America

* These authors contributed equally to this work.

${ }^{\dagger}$ Corresponding author: M. Salmerón-Sánchez, masalsan@,fis.upv.es

Abstract Fibronectin (FN) assembles into fibrillar networks by cells through an integrin-dependent mechanism. We have recently shown that simple FN adsorption onto poly(ethyl acrylate) surfaces (PEA), but not control polymer (poly(methyl acrylate), PMA), also triggered FN organization into a physiological fibrillar network. FN fibrils exhibited enhanced biological activities in terms of myogenic differentiation compared to individual FN molecules. In the present study, we investigate the influence of topological cues on the material-driven FN assembly and the myogenic differentiation process. Aligned and random electrospun fibers were prepared. While FN fibrils assembled on the PEA fibers as they do on the smooth surface, the characteristic distribution of globular FN molecules observed on flat PMA transformed into nonconnected FN fibrils on electrospun PMA, which significantly enhanced cell differentiation. The direct relationship between the fibrillar organization of FN at the material interface and the myogenic process was further assessed by preparing FN gradients on smooth PEA and PMA films. Isolated FN molecules observed at one edge of the substrate gradually interconnected with each other, eventually forming a fully developed network of FN fibrils on PEA. In contrast, FN adopted a globular-like conformation along the entire length of the PMA surface, and the FN gradient consisted only of increased density of adsorbed FN. Correspondingly, the percentage of differentiated cells increased monotonically along the FN gradient on PEA but not on PMA. This work demonstrates an interplay between material chemistry and topology in modulating material-driven FN fibrillogenesis and cell differentiation. 


\section{INTRODUCTION}

Fibronectin (FN) is a core glycoprotein $(\sim 220 \mathrm{kDa})$ of the extracellular matrix (ECM). It is synthesized by adherent cells, which afterwards assemble it into a fibrillar network through an integrin-dependent contractile mechanism [1,2]. Integrin-FN interactions promote a step-wise process that gives rise to both conformational changes of the FN molecule and the organization of the actin cytoskeleton. Conformational changes of FN induce the exposure of intermolecular binding sites that facilitate the assembly of a FN fibrillar matrix, so-called cell-mediated fibrillogenesis [3]. The thickness of the FN matrix fibrils ranges from 10 to $1000 \mathrm{~nm}$ in diameter and these fibrils consist of a few to hundred of FN molecules across. FN fibril assembly involves the $70 \mathrm{kDa}$ aminoterminal domain of $\mathrm{FN}$, through binding of $\mathrm{I}_{1-5}$ either to $\mathrm{III}_{1-2}$ or $\mathrm{III}_{12-14}$ domains [4].

The need for controllable and reproducible in vitro models of FN networks and for new synthetic materials able to serve as bio-inspired scaffolds for tissue engineering has driven the efforts for the identification of cell-free routes able to induce FN fibrillogenesis. These routes are based on the assumption that unfolding of soluble FN dimers from their globular conformation is needed for FN-FN interactions to occur, leading eventually to FN polymerization and fibril formation. The methods described so far include (i) addition of reducing [5] or oxidizing [6] agents to the protein solution; (ii) the use of denaturing [7], cationic [8] or anionic [9] compounds; (iii) the use of peptidic FN fragments [10]; and (iv) force-based assembly, via application of mechanical tension $[11,12]$ or shear forces [13-15].

We have recently shown that adsorption of individual FN molecules onto particular surface chemistries induces exposure of self-assembly sites to drive FN fibril assembly and identified poly(ethyl acrylate) (PEA) as a potential surface chemistry to generate FN fibrils, giving rise to a new process which we refer to as material-driven FN 
fibrillogenesis $[16,17,18]$. This work further investigates the biological activity of the material-driven FN network assembled on substrates that provide topological cues, evaluated by examining the myogenic differentiation process [19]. To do that, materialdriven FN fibrillogenesis was performed on random and aligned electrospun fibers, as well as on a gradient of FN fibrils (ranging from individual isolated FN molecules to the well-assembled FN network). Poly(methyl acrylate) (PMA) was used as a control polymer, since PMA surfaces do not promote FN fibrillogenesis [18].

The electrospinning technique allows for the production of very thin fibers with very large specific surface areas. The technique has gained importance in recent years for biomedical applications, such as tissue engineering, drug release, wound dressing, and enzyme immobilization [20,21]. Also, aligned electrospun fibers promote the orientation and differentiation of cells in the direction of the fibers [17,22,23]. Moreover, in our case, their use is biologically relevant since muscle consists of aligned myofibers, and, thus, not only the adequate differentiation of myoblasts, but also their alignment is important to achieve muscle regeneration. As a matter of fact, cell alignment is a prerequisite for the formation of contractile myotubes [24]. In vitro alignment approaches have thus become a usual method in tissue engineering, including the production of aligned micropatterned surfaces [25-26], microcontact printing of proteins [27] and aligned fibers [28]. Most of these studies focus on the influence of pattern size (from 3 to $100 \mu \mathrm{m}$ ) on cell alignment and morphology, with no emphasis on cell differentiation.

On the other hand, the preparation of gradients of properties allows one to investigate, on the same substrate, cellular responses, including migration and differentiation, to a continuous range of stimuli coming from the surface [29]. 
The aim of this work is to investigate the combined effect of topological cues and the material-driven FN network on myoblast differentiation, by modulating (i) surface topography with electrospun fibers and (ii) the variation of $\mathrm{FN}$-fibrillogenesis establishing FN gradients at the material interface. Specifically, we aim at investigating the role of the electrospun fibers (topography, curvature) on the material-driven FN network, to correlate the state of the layer of FN at the cell-material interface with cell differentiation. Also, the comparison between a gradient of FN density vs a gradient of FN-fibrillogenesis is intended to be correlate with the myogenic differentiation process.

\section{MATERIALS AND METHODS}

\subsection{Materials}

Polymer sheets were obtained by radical polymerization of a solution of the corresponding alkyl acrylate, i.e., methyl (MA) and ethyl (EA), (Sigma-Aldrich, Steinheim, Germany) using $1 \mathrm{wt} \%$ benzoin ( $98 \%$ pure, Scharlau, Barcelona, Spain) as photoinitiator. The polymerization was carried out up to limiting conversion. After polymerization, low molecular-mass substances were extracted from the material by drying in vacuum to constant weight.

\subsection{Spin coating}

Thin films were prepared by making use of a spin-coater (Brewer Science, Rolla, USA). Each one of the synthesized polymers was dissolved in toluene at a concentration of 5 $\mathrm{wt} \%$. Spin casting was performed on $15 \times 15 \mathrm{~mm}^{2}$ glass coverslips at $2000 \mathrm{rpm}$ for $30 \mathrm{~s}$. Samples were dried under vacuum at $60^{\circ} \mathrm{C}$ before use. For cell culture experiments samples were sterilized by UV exposure for $30 \mathrm{~min}$. 


\subsection{Electrospinning}

PEA and PMA were dissolved in hexafluoroisopropanol (HFIP, Sigma) at $20 \mathrm{mg} / \mathrm{mL}$ and loaded into a $1 \mathrm{~mL}$ syringe with an internal diameter of $5 \mathrm{~mm}$. Polymer solutions were electrospun at a constant feed rate of $900 \mu \mathrm{L} / \mathrm{h}$ using a programmable syringe pump (New Era Pump Systems, Wantagh, NY, USA) with a voltage of $12.5 \mathrm{kV}$ (Glassman High Voltage, High Bridge, NJ, USA) and a collector distance of $20 \mathrm{~cm}$. Randomly electrospun fibers were collected on glass covers placed on grounded aluminum foil (thereafter referred to as PMAr and PEAr, respectively). Aligned fibers were obtained under the same conditions, but the polymer solutions were electrospun onto a rotating drum (rotating at $900 \mathrm{rpm}$, equivalent to a linear speed of $337.5 \mathrm{~cm} / \mathrm{s}$ ) where glass coverslips were stuck (thereafter referred to as PMAa and PEAa, respectively). Before cell culture experiments, samples were sterilized by UV exposure for $30 \mathrm{~min}$.

\subsection{Scanning Electron Microscopy}

The electrospun fibers were characterized by a scanning electron microscope (SEM) JEOL JSM 6300 (JEOL Ltd., Tokyo, Japan) at $15 \mathrm{kV}$. In order to obtain quantitative data about fiber anisotropy, SEM data was processed using Image $\mathrm{J}$ software following an already described procedure [30]. Shortly thereafter, Fourier-Fast transform of the squared picture was realized, and after a counter-clockwise rotation of $90^{\circ}$ (in order to recover original fiber direction modified by the Fourier Transform) a radial projection of pixel intensity was determined using an external plug-in developed by O'Connell [31]. Normalized values (with respect to maximum intensity) are presented.

\subsection{Atomic Force Microscopy}


AFM was performed in a NanoScope III from Digital Instruments (Santa Barbara, CA) operating in the tapping mode; the Nanoscope 5.30r2 software version was used for image processing and analysis. Si-cantilevers from Veeco (Manchester, UK) were used with force constant of $2.8 \mathrm{~N} / \mathrm{m}$ and resonance frequency of $75 \mathrm{kHz}$. The phase signal was set to zero at a frequency $5-10 \%$ lower than the resonance one. Drive amplitude was $200 \mathrm{mV}$ and the amplitude setpoint (Asp) was $1.4 \mathrm{~V}$. The ratio between the amplitude setpoint and the free amplitude (Asp/A0) was kept equal to 0.7.

\subsection{Protein adsorption}

FN from human plasma (Invitrogen) was adsorbed on the different substrates by immersing the material sheets in FN solutions of concentration $20 \mu \mathrm{g} / \mathrm{mL}$ in Dubelcco's Phosphate Saline Buffer (DPBS). After adsorption, samples were rinsed in DPBS to eliminate the non-adsorbed protein. AFM was performed in the tapping mode immediately after sample preparation. Height, phase and amplitude magnitudes were recorded simultaneously for each image.

\subsection{Preparation of FN gradients}

For the preparation of FN gradients on thin films of PEA and PMA (thereafter referred to as gPEA and gPMA, respectively) a dip-coater device (KSV-DC Small vessel, KSVNIMA, Finland) was used. The squared samples were immersed up to a maximum depth of $10 \mathrm{~mm}$ at the highest possible velocity $(100 \mathrm{~mm} / \mathrm{min})$ in a $\mathrm{FN}$ solution $(10$ $\mu \mathrm{g} / \mathrm{mL}$ in DPBS) and pulled out at a controlled velocity of $6.7 \mathrm{~mm} / \mathrm{min}$, which corresponds to a maximum immersion time of $90 \mathrm{~s}$ (Fig. 5a). After adsorption, samples were rinsed in DPBS to eliminate the non-adsorbed protein. AFM was performed in the tapping mode immediately after sample preparation. 


\subsection{Cell culture}

Murine $\mathrm{C} 2 \mathrm{C} 12$ myoblasts were obtained from ATCC. Cells were maintained in Dulbecco's Modified Eagle Medium (DMEM) supplemented with 20\% fetal bovine serum (Fisher) and 1\% penicillin-streptomycin (Lonza) and passaged twice a week using standard techniques. Prior to seeding on substrates, electrospun fibers were coated with FN (Invitrogen) at $20 \mu \mathrm{g} / \mathrm{mL}$ for $1 \mathrm{~h}$ at room temperature, while FN gradients were prepared as previously described. Then $\mathrm{C} 2 \mathrm{C} 12$ cells were seeded at 20,000 cells $/ \mathrm{cm}^{2}$ on the different surfaces in DMEM supplemented with 1\% penicillin-streptomycin and 1\% insulin-transferrin-selenium-X (Invitrogen) to induce myogenic differentiation.

\subsection{Myogenic differentiation}

C2C12 cells were cultured on FN-coated materials for 4 days under differentiation conditions and immunostained for sarcomeric myosin. Briefly, cultures were fixed in $70 \%$ ethanol $/ 37 \%$ formaldehyde/glacial acetic acid (20:2:1) and then blocked in 5\%

goat serum in DPBS for $1 \mathrm{~h}$. Samples were sequentially incubated in MF-20 mouse antibody (Developmental Studies Hybridoma Bank, University of Iowa, USA) and antimouse Cy3-conjugated secondary antibody (Jackson Immunoresearch). Finally, samples were washed before being mounted in Vectashield containing DAPI (Atom). Cultures were scored by the percentage of cells positive for sarcomeric myosin using in-house image analysis software developed under MATLAB R2006a (The MathWorks, Inc., Natick, MA) [18]. Anisotropy in cell distribution was evaluated in the same way described for the anisotropy in fiber distribution, using the images of sarcomeric myosin staining; values were normalized and an arbitrary offset was applied in order to avoid overlapping of the different curves. 


\subsection{Statistical Analysis}

Results are shown as average \pm standard deviation. All experiments were performed at least three times in triplicate unless otherwise noted. Results were analyzed by one-way ANOVA. If treatment level differences were determined to be significant, pair-wise comparisons were performed.

\section{RESULTS AND DISCUSSION}

\subsection{Material-driven fibronectin fibrillogenesis}

Poly(ethyl acrylate) (PEA) and poly (methyl acrylate) (PMA) differ in one single carbon in the side chain (Fig. 1a), show similar wettability (WCA $\sim 80^{\circ}$ ) and total amount of adsorbed FN (surface density $\sim 450 \mathrm{ng} / \mathrm{cm}^{2}$ ) [18]. By contrast, the organization of FN after adsorption from solutions of the same concentration onto thin films of PEA and PMA differs: while interconnected network-like fibrils are found on PEA, only dispersed FN molecules are observed on PMA (Fig. 1b). The spontaneous formation of a FN-network on PEA was described to be a time- and concentrationdependent process, as the organization of interconnected FN fibrils occurs within minutes of adsorption [32] from solutions of different FN concentrations [17].

The fibrillar-like organization of FN upon passive adsorption on PEA was termed material-driven fibrillogenesis, since the assembled FN fibrils on PEA share some similarities with the physiologically cell-assembled FN matrices. In particular, $\mathrm{I}_{1-5}$ repeats located within the $70 \mathrm{kDa}$ amino-terminal regions are essential for cell-mediated FN assembly [33]. This domain is not accessible in the folded, compact structure of FN in solution and a conformational change of the molecule is mandatory for physiological matrix assembly to occur [3]. We have recently found that for the material-driven 
fibrillogenesis to occur, the $70 \mathrm{kDa}$ amino-terminal region of $\mathrm{FN}$ is absolutely required. Addition of a recombinant $70 \mathrm{kDa}$ fragment during FN adsorption on PEA completely blocked the organization of $\mathrm{FN}$ at the material interface, giving rise to discrete molecular aggregates resembling the FN adsorbed on PMA [18].

More important is that, because fibrillar structure recapitulates the native structure of FN matrices, the material-driven FN fibrils displayed enhanced biological activity, as evaluated by examining the myogenic differentiation process [19]. Sarcomeric myosin expression and cell bipolar alignment and fusion into myotubes, markers of myogenesis, were significantly higher on the substrate-induced FN network on PEA than on PMA, where no fibrillar organization occurs [18].

\subsection{Effect of topological cues: Myoblast behavior on electrospun fibers}

We next examined the role of topographical cues coming from elecrospun fibers on the formation of the FN fibrils on both PEA and PMA, as well as their influence on the myogenic differentiation process.

Homogenous straight fibers of PEA and PMA were obtained via electrospinning (Fig. 2) and no beading was observed. Dimensions of the fibers were similar in all cases, ranging from $2.2 \pm 0.9 \mu \mathrm{m}$ for PMAr to $3.9 \pm 1.4 \mu \mathrm{m}$ for PEAa. Similarly, the average distance between fibers was similar for the aligned fibers $(3.3 \pm 1.6$ for PEAa and $2.3 \pm 1$ for PMAa). Therefore, the topology is equivalent for both PMA and PEA electrospun substrates, and hence differences in cell behavior between PEA and PMA fibers cannot be attributed to any significant differences between the topology of the electrospun polymers. Fiber orientation quantified by image analysis is depicted in Fig. 2. As expected, random fibers show no preferential direction, while aligned fibers display a characteristic peak as a consequence of a highly anisotropic organization. 
AFM was used to investigate FN distribution on the electrospun PEA and PMA fibers (Fig. 3). Electrospun PEA drives FN organization into fibrillar networks similarly to the organization on flat PEA surfaces [17]. Consequently, a network of FN fibrils along PEA fibers was observed. By contrast, FN distribution on electrospun PMA fibers differs from the one observed on flat PMA: rather than globular aggregates, extended FN molecules can be seen on PMA fibers, resembling the distribution of FN on PEA samples during the initial assembly of the FN fibrils, where the formation of the FN network is not complete yet [32]. As a matter of fact, it was previously reported that electrospun polymer fibers are able to alter the distribution of FN compared to the solvent cast smooth surface, promoting the formation of organized FN fibrils aligned along the length of the fiber [34].

The myogenic differentiation process was quantified on the electrospun polymers by immunostaining for sarcomeric myosin. Similar differentiation levels were found on random and aligned fibers of every polymer (Fig. 4). As expected, the substrate-induced FN network on PEA fibers enhanced differentiation compared to collagen type I, which is considered to be the standard substrate for myogenic differentiation [18]. By contrast, myoblast differentiation on electrospun PMA increases dramatically compared to collagen type I (Fig. 4) and smooth PMA surfaces [18]. We attribute this result to the differences in FN structure between on the electrospun fibers and the smooth surface. Dispersed FN molecules were obtained on flat PMA [18], whereas an incipient network formation of FN fibrils, which are known to enhance myogenic differentiation, was assembled on the electrospun polymer (Fig. 3).

Regardless the underlying chemistry, electrospun fibers trigger cell orientation as observed in the fluorescence images and quantified by image analysis (Fig. 4a and 4b). Differentiated myoblasts remain isotropically distributed on collagen type I. Clusters of 
cells with preferred orientations are observed on random fibers (PEAr and PMAr), as it is revealed by image analysis with a broad peak resulting from the superposition of the intensity found for every cluster of cells aligned in a different direction (Fig. 4c). In contrast, myoblasts aligned along the direction of the fibers on both PEAa and PMAa and image analysis show a characteristic orientation peak (intensity falls to baseline levels when the angle between the cell and the fiber direction is above $30^{\circ}$ ).

Even though myoblast alignment is mandatory for their fusion into myotubes [35], the orientation of myoblasts in the direction of the aligned fibers did not cause any enhancement in the differentiation levels on PEAa or PMAa compared to PEAr or PMAr (Fig. 4b). This results is probably related to limited cell-cell contact at the poles of the aligned cells on our surfaces, which is needed to enhance myogenic differentiation via myotube formation [24,36]. Concordantly, other authors have shown no enhancement in myoblast differentiation on micropatterned aligned channels with controlled chemistry [37]. Nevertheless, it cannot be discounted that the interplay between cell alignment and differentiation might not be detected via sarcomeric myosin staining, and other subtle effects such as modified cytoskeletal organization or increased cell contractility $[38,39]$ might occur. Further studies are needed to investigate the role of substrate-induced cell alignment on myogenesis.

\subsection{Myoblast behavior on FN gradients}

The effect of FN fibrillogenesis on myoblast differentiation was further studied by making use of gradients of adsorbed FN onto flat substrates of PEA (gPEA) and PMA (gPMA). FN gradients were obtained by pulling out the samples from a FN solution of concentration $10 \mu \mathrm{g} / \mathrm{ml}$ at a controlled velocity. In the case of PEA, AFM revealed the progressive formation of a FN network as the adsorption time increased: the isolated FN 
aggregates observed at one end of the substrate gradually interconnected with each other, eventually forming a fully developed network of FN fibrils (Fig. 5b). In the case of PMA, no fibril formation was detected, as FN adopted a globular-like conformation along the entire length of the substrate; increasing the adsorption time only increased the amount of adsorbed FN but did not yield any fibrillogenesis (Fig. 5b). It can also be appreciated that at the low pull-out velocity and FN concentration used to produce these gradients, no shear-stress induced FN fibrillogenesis was seen on either substrate [40].

The effect of the different FN conformation and distribution along the two types of gradient (gPEA and gPMA) on cell differentiation was evaluated by means of sarcomeric myosin immunodetection (Fig. 6a). The FN gradient on PMA sustained homogeneous differentiation along its length; indeed, no statistically significant differences were found between the degrees of differentiation at different positions along the sample (Fig. 6b). On the other hand, the assembly of FN fibrils that accompanied the increasing amount of adsorbed FN on PEA led to a monotonic increase in the differentiation degree along the gradient. A statistically significant enhancement in myogenic differentiation was observed at the end of the gradient, where the FN network was fully developed (Fig. 6b).

Both in gPEA and gPMA cell density increased monotonically alongside the FN gradient, with the differences more significant for PEA than PMA (Fig. 6b). Considering that the surface density of $\mathrm{C} 2 \mathrm{C} 12$ cells is not sensitive to the amount or distribution of FN adsorbed on either PEA or PMA [18], our data suggest that cells initially adhered uniformly along the FN gradient and then migrated in response to the biochemical gradient of signals provided by the formation of the FN gradient. Former studies have established that FN gradients induce haptotaxis for different cell types [4145]; concretely, FN has been suggested to promote myoblast migration [46]. Indeed, 
migration was more prominent along PEA, where FN adopts a more biologically active, physiological-like, distribution compared to PMA [18], consistent with the diminished differentiation with respect to PMA (Fig. 6b). Cell migration is in fact inversely correlated with proliferation and differentiation of myoblasts [47-48].

Taken together, these data further demonstrate the role of material-driven fibrillogenesis on myogenic differentiation. A gradient wherein the sole amount of FN was varied, but where the globular-like conformation of the protein was maintained (i.e., gPMA), did not yield any differential effect on myoblast differentiation and, still, supported cell migration. On the other hand, a gradient characterized by a gradual formation of FN fibrils (i.e., gPEA) led to cell migration and to a gradient of myogenic differentiation. We attributed the increases in cell differentiation along this gradient to gradual formation of a network of FN fibrils [18] and a progressively higher cell density [49]. To our knowledge, this is the first report where the effect of a protein gradient on myogenic differentiation is investigated, demonstrating the potential of this kind of biochemical cue to control and drive cell differentiation. Moreover, this study further emphasizes the role of protein conformation in cell-material interaction, since a FN gradient on a different material (PMA) does not induce any significant effect on myoblast differentiation.

\section{CONCLUSIONS}

We have investigated the relationship between material-driven FN fibrillogenesis and topological cues in the myogenic differentiation process. Topographical signals coming from aligned electrospun fibers orientated cells without modifying cell differentiation compared to random fibers of the same polymer. Strikingly, the distribution of FN on the PMA fibers was altered compared to the smooth surface, with the formation of 
initial FN fibrils that enhanced cell differentiation, supporting the role of the materialdriven FN assembly on the myogenic process. Likewise, a gradient of globular FN molecules on PMA did not alter cell differentiation, but the increasing formation of a FN network on PEA led to higher levels of myogenesis.

\section{Acknowledgements}

The support of the Spanish Ministry of Science and Innovation through project MAT2009-14440-C02-01 is acknowledged. CIBER-BBN is an initiative funded by the VI National R\&D\&i Plan 2008-2011, Iniciativa Ingenio 2010, Consolider Program, CIBER Actions and financed by the Instituto de Salud Carlos III with assistance from the European Regional Development Fund. This work was supported by funds for research in the field of Regenerative Medicine through the collaboration agreement from the Conselleria de Sanidad (Generalitat Valenciana), and the Instituto de Salud Carlos III.

\section{References}

1. Singh P, Carraher C, Schwarzbauer JE. Assembly of Fibronectin Extracellular Matrix. Ann Rev Cell Dev Biol. 2010;26: 397-419.

2. Hynes RO. Fibronectins. Springer Series in Molecular Biology, Springer New York, 1990.

3. Mao Y, Schwarzbauer JE. FN fibrillogenesis, a cell-mediated matrix assembly process. Matrix Biol. 2005;24: 389-399.

4. Geiger B, Bershadsky A, Pankov R, Yamada KM. Transmembrane extracellular matrix - cytoskeleton crosstalk. Nat Rev Mol Cell Biol. 2001;2: 793-805.

5. Sakai K, Fujii T, Hayashi T. Cell-free formation of disulfide-bonded multimer from isolated plasma fibronectin in the presence of a low concentration of $\mathrm{SH}$ reagent under a physiological condition. J Biochem.1994;115: 415-421. 
6. Vartio T. Disulfide-bonded polymerization of plasma fibronectin in the presence of metal ions. J Biol Chem. 1986;261: 9433-9437.

7. Mosher DF, Johnson RB. In vitro formation of disulfide-bonded fibronectin multimers. $\mathbf{J}$ Biol Chem. 1983;258: 6595-601.

8. Vuento M, Vartio T, Saraste M, von Bonsdorff $\mathrm{CH}$, Vaheri A. Spontaneous and polyamine-induced formation of filamentous polymers from soluble fibronectin. Eur $\mathrm{J}$ Biochem.1980;105: 33-42.

9. Richter H, Wendt C, Hörmann H. Aggregation and Fibril Formation of Plasma Fibronectin by Heparin. Biol Chem Hoppe-Seyler.1985;366: 509-514.

10. Morla A, Zhang Z, Ruoslahti E. Superfibronectin is a functionally distinct form of fibronectin. Nature 1994;367: 193-196.

11. Baneyx G, Vogel V. Self-assembly of fibronectin into fibrillar networks underneath dipalmitoyl phosphatidylcholine monolayers: role of lipid matrix and tensile forces. Proc Natl Acad Sci. 1999;96: 12518-12523.

12. Ulmer J, Geiger B, Spatz JP. Force-induced fibronectin fibrillogenesis in vitro. Soft Matter 2008;4: 1998-2007.

13. Brown RA, Blunn GW, Ejim OS. Preparation of orientated fibrous mats from fibronectin: composition and stability. Biomaterials. 1994;15: 457-464.

14. Little WC, Smith ML, Ebneter U, Vogel V. Assay to mechanically tune and optically probe fibrillar fibronectin conformations from fully relaxed to breakage. Matrix Biol. 2008;27: 451-461.

15. Klotzsch E, Smith ML, Kubow KE, Muntwyler S, Little WC, Beyeler F, Gourdon D, Nelson BJ, Vogel V. Fibronectin forms the most extensible biological fibers displaying switchable force-exposed cryptic binding sites. Proc Natl Acad Sci. 2009;106: 1826718272.

16. Rico P, Rodríguez Hernández JC, Moratal D, Altankov G, Monleón Pradas M, Salmerón-Sánchez M. Substrate-induced assembly of fibronectin into networks: Influence of surface chemistry and effect on osteoblast adhesion. Tiss Eng Part A 2009; 15: 3271-3281. 
17. Gugutkov D, González-García C, Rodríguez Hernández JC, Altankov G, SalmerónSánchez M. Biological activity of the substrate-induced FN network: Insight into the third dimension through electrospun fibers. Langmuir. 2009;25: 10893-10900.

18. Salmerón-Sánchez M, Rico P, Moratal D, Lee T, Schwarzbauer J, García AJ. Role of material-driven fibronectin fibrillogenesis in cell differentiation. Biomaterials 2011;32: 2099-2115.

19. Sabourin LA, Rudnicki MA. The molecular regulation of myogenesis. Clin Genet 2000;57: 16-25.

20. Agarwal S, Wendorff J, Greiner A. Use of electrospinning technique for biomedical applications. Polymer 2008;49: 5603-5621.

21. Sill TJS, von Recum HA. Electrospinning: Applications in drug delivery and tissue engineering. Biomaterials 2008;29: 1989-2006.

22. Huber A, Pickett A, Shakesheff KM. Reconstruction of spatially orientated myotubes in vitro using electrospun, parallel microfibre arrays. Eur Cells Mater. 2007;14: 56-63

23. Jun I, Jeong S, Shin H. The stimulation of myoblast differentiation by electrically conductive sub-micron fibers. Biomaterials 2009;30: 2038-2047.

24. Clark P, Dunn GA, Knibbs A, Peckham M. Alignment of myoblasts on ultrafine gratings inhibits fusion in vitro. Int J Biochem Cell Biol. 2002; 34:816-825

25. Lam MT, Sim S, Zhu X, Takayama S. The effect of continuous wavy micropatterns on silicone substrates on the alignment of skeletal muscle myoblasts and myotubes. Biomaterials. 2007;27: 4340-4347.

26. Altomare L, Gadegaard N, Visai L, Tanzi MC, Farè S. Biodegradable microgrooved polymeric surfaces obtained by photolithographyfor skeletal muscle cell orientation and myotube development. Acta Biomaterialia 2010;6: 1948-1957.

27. Altomare L, Riehle M, Gadegaard N, Tanzi MC, Farè S. Microcontact printing of fibronectin on a biodegradable polymeric surface for skeletal muscle cell orientation. Int J Artif Organs. 2010;33: 535-543.

28. Neumann T, Hauschka SD, Sanders JE. Tissue Engineering of Skeletal Muscle Using Polymer Fiber Arrays. Tissue Eng. 2003;9: 995-1003. 
29. Tse JR, Engler A. Stiffness Gradients Mimicking In Vivo Tissue Variation Regulate Mesenchymal Stem Cell Fate. Plos One 2011;6: e15978.

30. Gómez-Tejedor JA, Van Overberghe N, Rico P, Gómez Ribelles JL. Assessment of the parameters influencing the fiber characteristics of electrospun poly(ethyl methacrylate) membranes. Eur Polym J. 2011;47: 119-129.

31. O’Connell B. Oval Profile Plot. Research Services Branch, National Institute of Mental Health, National Institute of Neurological Disorders and Stroke. Available from: http://rsbweb.nih.gov/ij/plugins/oval-profile.html

32. Gugutkov D, Altankov G, Rodríguez Hernández JC, Monleón Pradas M, Salmerón Sánchez M. Fibronectin activity on substrates with controlled $-\mathrm{OH}$ density. Journal of Biomedical Materials Research 2010; A92: 322-331.

33. Schwarzbauer JE. Identification of FN sequences required for assembly of a fibrillar matrix. J Cell Biol 1991;113: 1463-1473.

34. Mukhatyar VJ, Salmerón-Sánchez M, Rudra S, Mukhopadaya S, Barker TH, García AJ, Bellamkonda RV. Role of fibronectin in topographical guidance of neurite extension on electrospun fibers. Biomaterials 2011; 32: 3958-3968.

35. Wakelam MJ. The fusion of myoblasts. Biochem J 1985; 228: 1-12.

36. Quach NL, Rando TA. Focal adhesion kinase is essential for costamerogenesis in cultured skeletal muscle cells. Developmental Biology 2006; 293: 38-52

37. Charest JL, García AJ, King WP. Myoblast alignment and differentiation on cell culture substrates with microscale topography and model chemistries. Biomaterials 2007; 28: 2202-2210

38. Berendse M, Grounds MD, Lloyd CM. Myoblast structure affects subsequent skeletal myotube morphology and sarcomere assembly. Experimental Cell Research 2003; 291: $435-450$.

39. Li B, Lin M, Tang Y, Wang B, Wang JHC. Novel functional assessment of the differentiation of micropatterned muscle cells. Journal of Biomechanics 2008; 41: $3349-3353$. 
40. Blunn GW, Brown RA. Production of artificial-oriented mats and strands from plasma fibronectin: a morphological study. Biomaterials. 1993;14: 743-748.

41. Smith JT, Tomfohr JK, Wells MC, Beebe TP, Kepler TB, Reichert WM. Measurement of cell migration on surface-bound fibronectin gradients. Langmuir 2004; 20:8279-86.

42. Smith JT, Elkin JT, Reichert WM. Directed cell migration on fibronectin gradients: effect of gradient slope. Exp Cell Res. 2006;312: 2424-2432.

43. Rhoads DS, Guan JL. Analysis of directional cell migration on defined FN gradients: role of intracellular signaling molecules. Exp Cell Res. 2007;313: 3859-3867.

44. Liu L, Ratner BD, Sage EH, Jiang S. Endothelial cell migration on surface-density gradients of fibronectin, VEGF, or both proteins. Langmuir. 2007;23: 11168-11173.

45. Shi J, Wang L, Zhang F, Li H, Lei L, Liu L, Chen Y. Incorporating protein gradient into electrospun nanofibres as scaffolds for tissue engineering. ACS Appl Mater Interfaces. 2010;2: 1025-1030.

46. Goetsch KP, Kallmeyer K, Niesler CU. Decorin modulates collagen I-stimulated, but not fibronectin-stimulated, migration of C2C12 myoblasts. Matrix Biol. 2011;30: 109117.

47. Bondesen BA, Jones KA, Glasgow WC, Pavlath GK. Inhibition of myoblast migration by prostacyclin is associated with enhanced cell fusion. FASEB J. 2007;21: 3338-3345.

48. Olguin HC, Santander C, Brandan E. Inhibition of myoblast migration via decorin expression is critical for normal skeletal muscle differentiation. Dev Biol. 2003;259: 209-224.

49. Konigsberg IR. Diffusion-mediated control of myoblast fusion. Dev Biol. 1971;26: $133-152$ 


\section{Captions to Figures}

Fig. 1 FN distribution on material substrates. (a) Chemical structure of the polymers PEA and PMA. (b) FN distribution on material substrates as obtained by AFM: dispersed aggregates on PMA and FN network on PEA after adsorption from a solution of concentration $10 \mu \mathrm{g} / \mathrm{mL}$

Fig. 2 Electrospun materials. Left column: representative SEM micrograph; central column: FFT of the SEM picture; right column: radial projection of the normalized pixel intensity of the FFT image. The scale bar is valid for every image.

Fig. 3 FN distribution on electrospun materials as obtained by AFM from a solution of concentration 20 $\mu \mathrm{g} / \mathrm{mL}$. FN networks are observed on both random and aligned PEA fibers. Non-interconnected FN fibrils are observed on PMA fibers.

Fig. 4 Myogenic differentiation on the different electrospun materials. (a) Fluorescence staining showing sarcomeric myosin-positive cells (green) and cell nuclei (red). (b) Myogenic differentiation as determined by the percentage of sarcomeric myosin-positive cells. Statistically significant differences (as determined by ANOVA) are indicated with * $(\mathrm{p}<0.05)$. (c) Orientation of differentiated cells as calculated by FFT and radial projection of the normalized pixel intensity. The scale bar is valid for every image.

Fig. 5 Gradients of adsorbed FN. (a) Sketch of the FN gradient on a PEA- or PMA-coated $15 \times 15 \mathrm{~mm}^{2}$ cover glass. (b) FN conformation along the gradient, as observed by the phase magnitude in AFM: on PEA FN gradually forms an interconnected network of fibrils, whilst on PMA no fibrillogenesis is observed, as FN maintains a globular conformation while the amount of adsorbed protein increases.

Fig. 6 Myogenic differentiation along the FN gradients. (a) Fluorescence staining showing sarcomeric myosin-positive cells (green) and cell nuclei (red) at different positions $(0,5$, and $10 \mathrm{~mm})$ along the two types of gradient (gradient of FN on PEA, gPEA, and on PMA, gPMA). (b) Myogenic differentiation (as determined by the percentage of sarcomeric myosin-positive cells) and cell density at different positions along the gradients. Statistically significant differences (as determined by ANOVA) are indicated with * $(\mathrm{p}<0.1), * *(p<0.05), \dagger(\mathrm{p}<0.005)$. The scale bar is valid for every image. 
Figure 1

a<smiles>CCCC(=O)OCC</smiles>

$\left(\mathrm{CH}_{2}-\mathrm{CH}\right)_{\bar{n}}$

b poly(ethyl acrylate), PEA<smiles>COC=O</smiles>

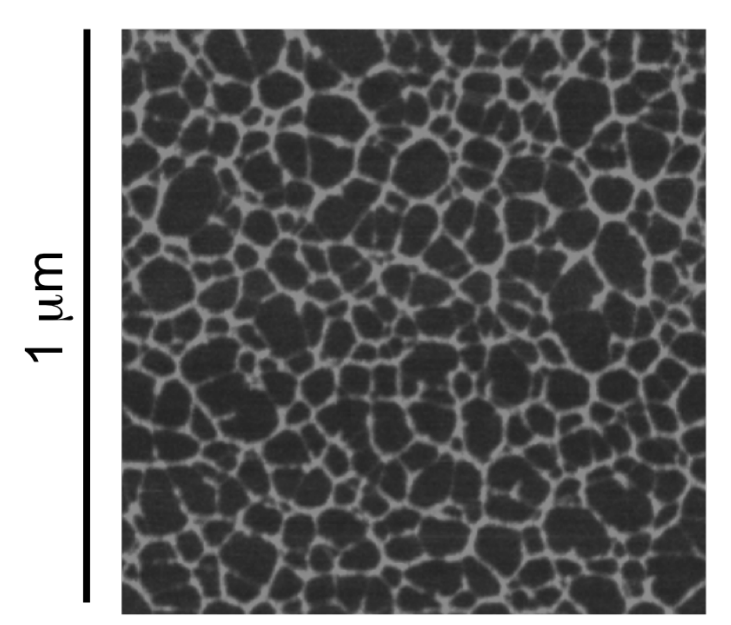

poly(methyl acrylate), PMA

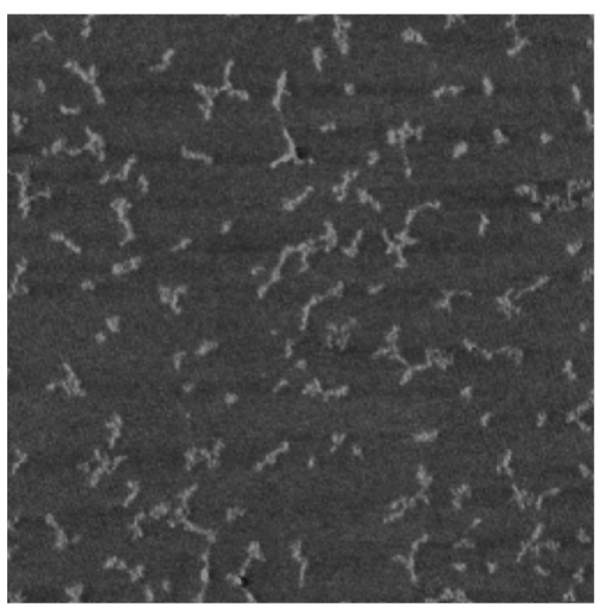


Figure 2
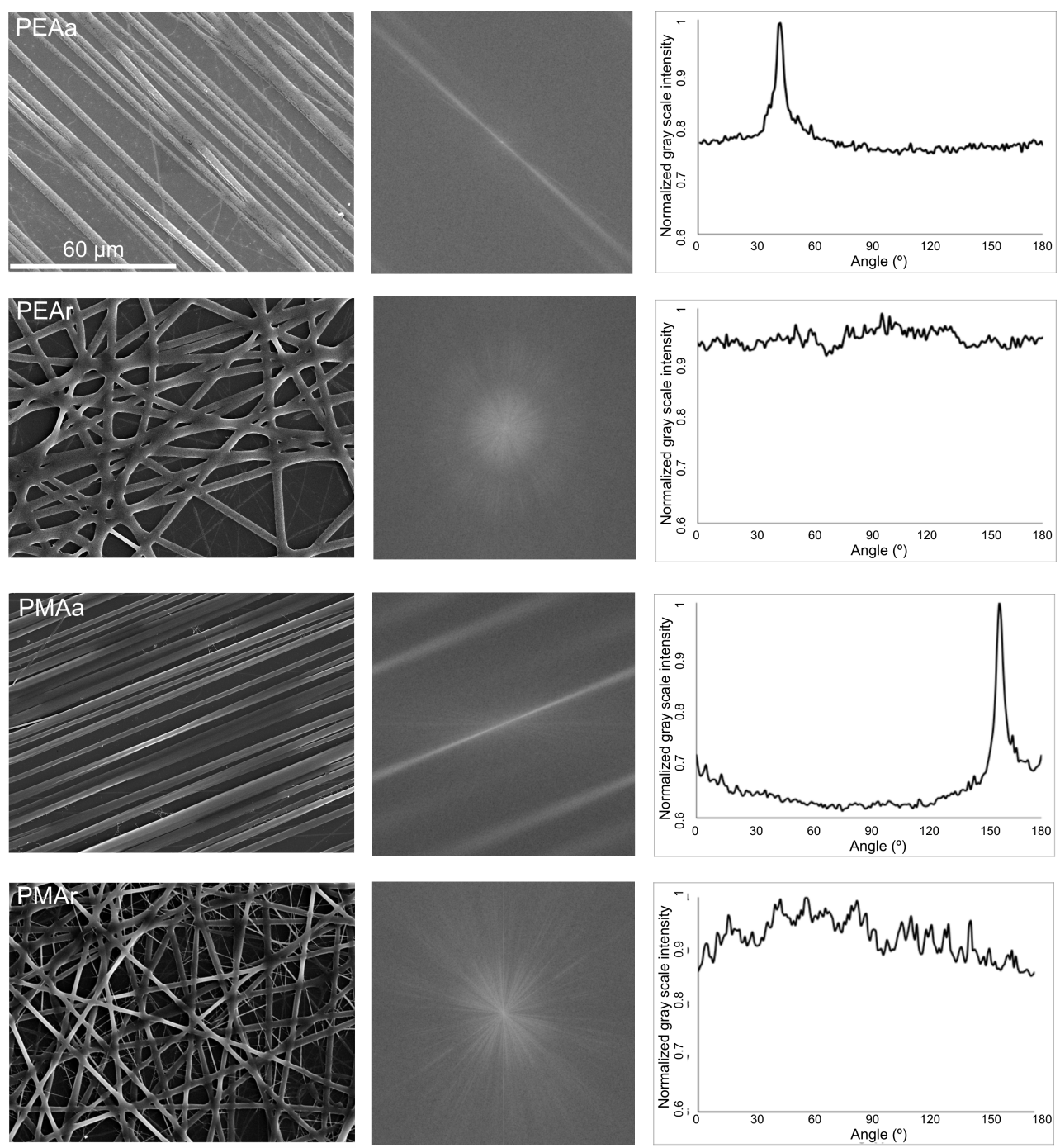
Figure 3

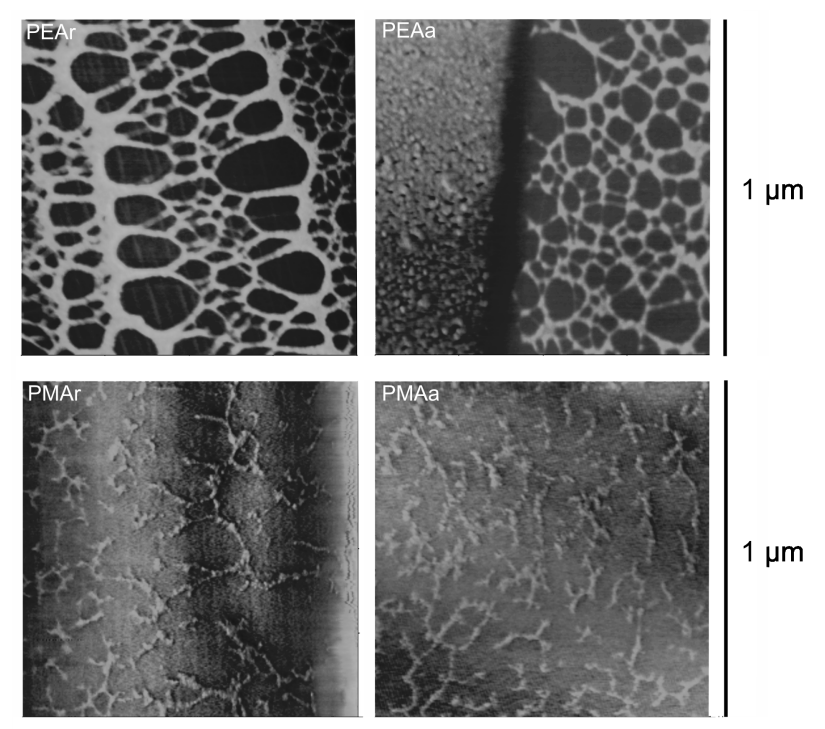


Figure 4

a

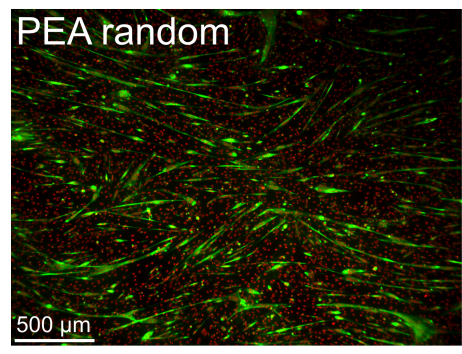

PMA random

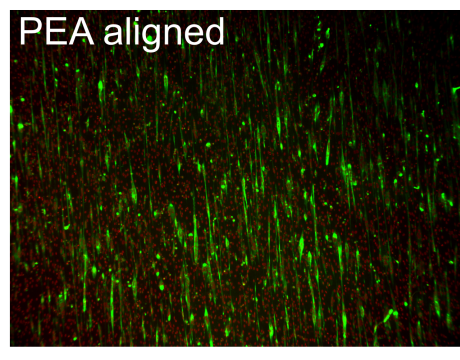

PMA aligned

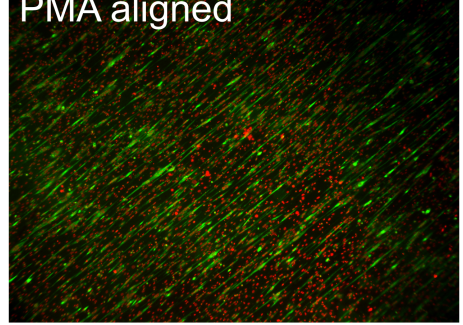

b
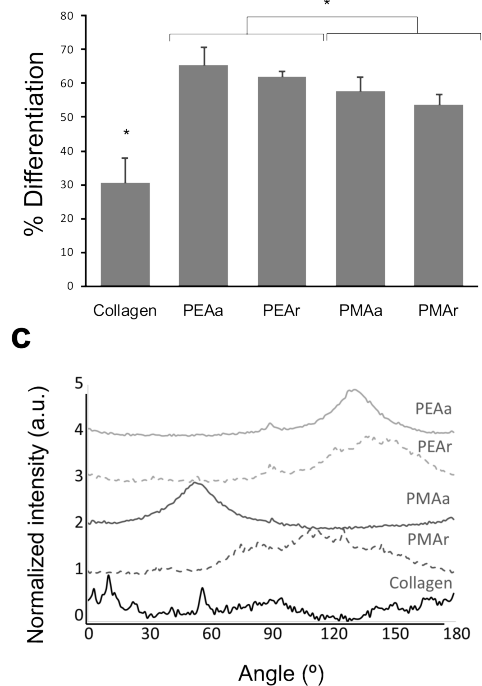
Figure 5

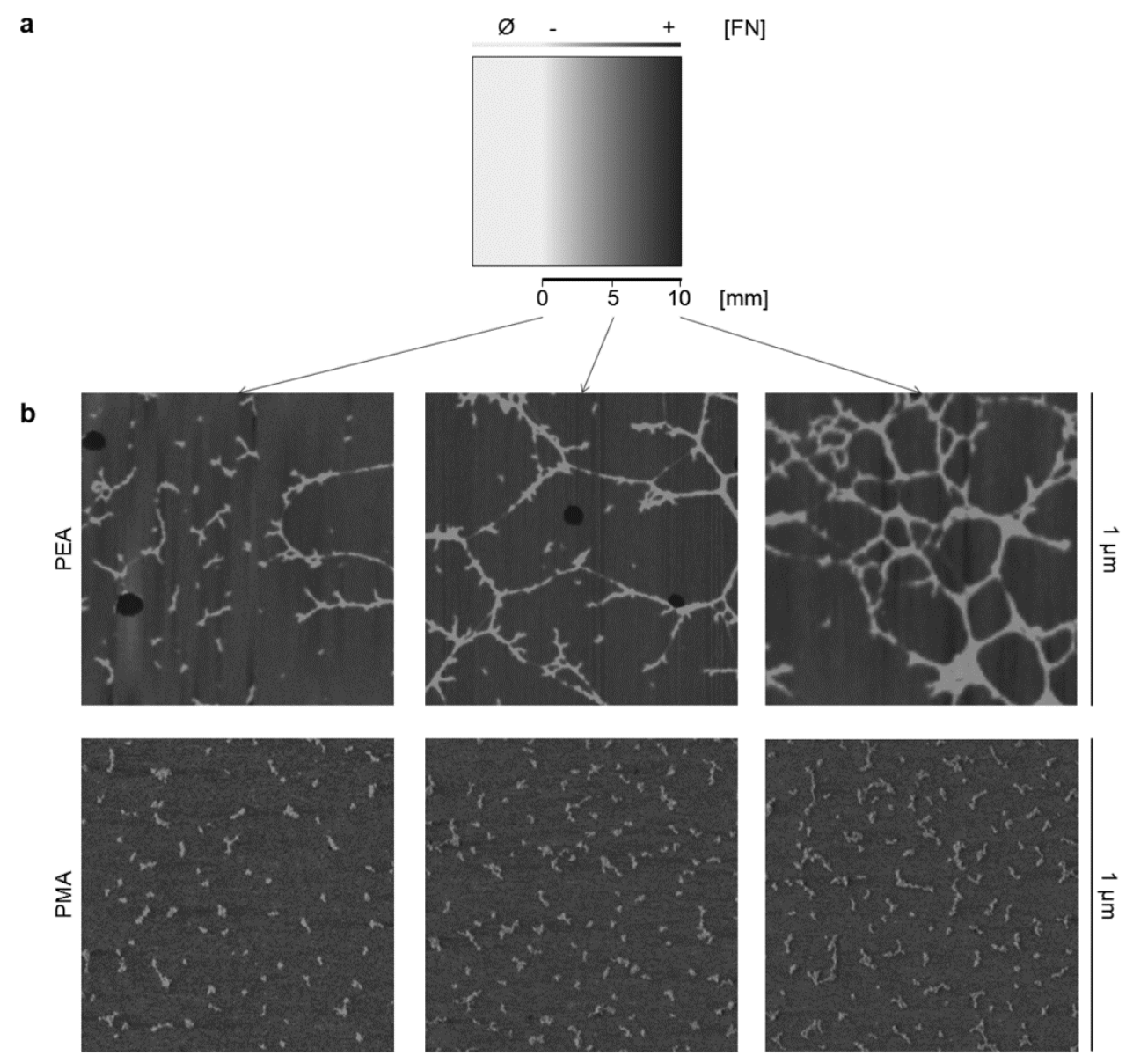


Figure 6
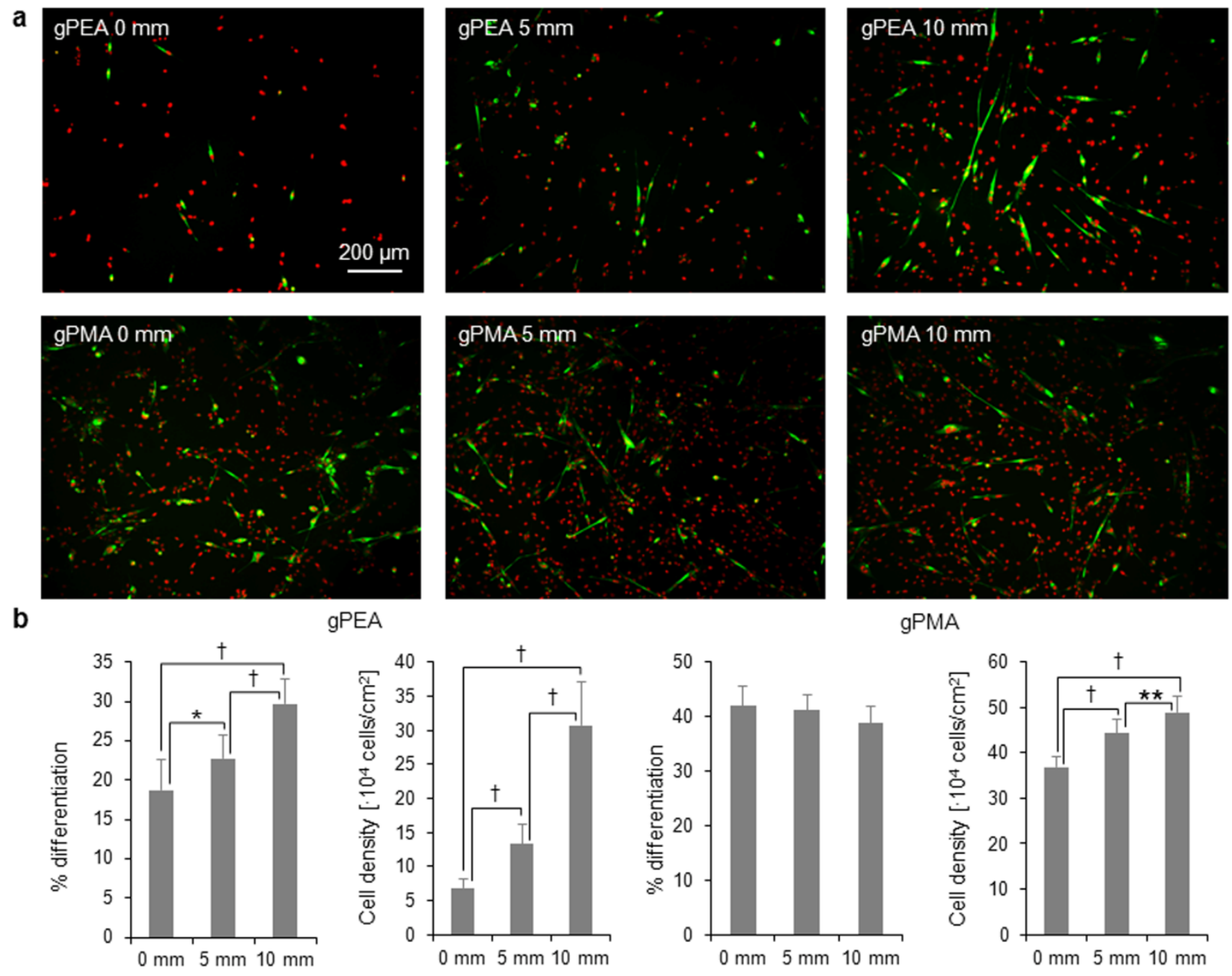